\title{
Assessing longitudinal and cross-sectional effects of age on adult obesity in an Iranian population: results from a large population-based cohort study
}

Mahsa Rafiee Alhossaini

Tarbiat Modares University Faculty of Medical Sciences

Anoshirvan Kazemnejad

Tarbiat Modares University Faculty of Medical Sciences

Farid Zayeri

Shaheed Beheshti University of Medical Sciences

Masoumeh Sadeghi ( $\nabla$ m_sadeghi@crc.mui.ac.ir)

Isfahan University of Medical Sciences https://orcid.org/0000-0001-7179-5558

Research article

Keywords: Obesity, Adult, Age, Longitudinal, Cross-sectional, Prevalence

Posted Date: March 29th, 2021

DOI: https://doi.org/10.21203/rs.3.rs-21244/v4

License: (c) (i) This work is licensed under a Creative Commons Attribution 4.0 International License.

Read Full License 


\section{Assessing longitudinal and cross-sectional effects of age on adult obesity in}

\section{an Iranian population: results from a large population-based cohort study}

Mahsa Rafiee Alhossaini ${ }^{1}$, Anoshirvan Kazemnejad ${ }^{1 *}$, Farid Zayeri ${ }^{2}$, Masoumeh Sadeghi ${ }^{3 *}$

${ }^{1}$ Department of Biostatistics, Faculty of Medical Sciences, Tarbiat Modares University, Tehran, Iran

${ }^{2}$ Proteomics Research Center and Department of Biostatistics, School of Allied Medical Sciences, Shahid Beheshti University of Medical Sciences, Tehran, Iran

${ }^{3}$ Cardiac Rehabilitation Research Center, Isfahan Cardiovascular Research Institute, Isfahan University of Medical Sciences, Isfahan, Iran, m_sadeghi@crc.mui.ac.ir

* Corresponding authors:

Prof. Anoshirvan Kazemnejad, Ph.D.

Department of Biostatistics, Faculty of Medical Sciences, Tarbiat Modares University, P.O Box 14115111, Tehran, Iran, Phone: +98 21 82883875, Email: kazem_an@modares.ac.ir

Prof. Masoumeh Sadeghi, MD.

Cardiac Rehabilitation Research Center, Isfahan Cardiovascular Research Institute, Isfahan University of Medical Sciences, P.O Box 58388994, Isfahan, Iran, Phone: +98 3136115210 , Email: m_sadeghi@crc.mui.ac.ir

Abstract word count: 343

Text word count: 4603 


\section{Abstract}

\section{Background}

Obesity is a significant risk factor for Noncommunicable diseases, and it is related to many adverse health consequences. The risk of obesity commonly changes with age, which is called a longitudinal or aging effect. Also, individuals born or enter to the study of the same age have similar living conditions that may influence their obesity risk in a particular way; this is a crosssectional effect. In the current study, an advanced statistical model is used to distinguish between longitudinal and cross-sectional effects of age on the risk of obesity for men and women.

\section{Methods}

Participants are a group of 6504 Iranian adults over 35 years of age in 2001, who live in the central region of Iran. They were followed up for 12 years in a large community-based study. Various medical indexes, including Body Mass Index, were collected in 2001, 2007, and 2013. The Marginal Logistic Regression model, which includes linear and quadratic effects of the Baseline Age and its difference with current age, is used.

\section{Results}

Between 2001 and 2013, the prevalence of obesity raised from 13\% to $18 \%$ in men and from $31 \%$ to $44 \%$ in women. The odds of obesity for women was approximately three times the odds of obesity for men on average adjusting for the age effects. Both cross-sectional and longitudinal effects of age were significantly associated with the odds ratio of obesity. There was a rise in the prevalence of obesity for individuals with Baseline Age 35 to 55 and a decline thereafter. Also, the odds ratio of obesity across one's life course, had about $3 \%$ increase, on average, by each year aging, regardless of the age at baseline.

\section{Conclusions}


The high rate of obesity and its fast growth is a serious public health issue among Iranians, especially in adults age 35-55, and women. In the present study, Baseline Age was more strongly associated with the risk of obesity than aging. Considering both cross-sectional and longitudinal effects of age, helps us to understand the effect of age on obesity better and to identify the related factors.

\section{Key Words:}

Obesity, Adult, Age, Longitudinal, Cross-sectional, Prevalence

\section{Background}

Noncommunicable Diseases (NCDs) account for more than $70 \%$ of early deaths all over the world $(1,2)$. Obesity, as a significant risk factor for NCDs, has an association with life expectancy reduction (3), and it raises the risk of metabolic diseases to a considerable extend(1). Also, obesity is associated with several adverse health consequences, and it is tied to an increased risk of conditions. Furthermore, obesity leads to a reduction in the quality of life, lack of employment, lower productivity, and inadequate social points. The World Obesity Federation and other organizations have defined obesity not only as a risk factor for several diseases but also as a chronic progressive disease (3). Obesity is defined as excessive fat accumulation that might have a negative effect on health by the World Health Organization (WHO) and is identified as Body Mass Index (BMI) over or equal to $30 \mathrm{~kg} / \mathrm{m}^{2}(4,5)$.

Obesity has an association with age, gender, household income, urbanization, and lifestyle (6). It is a chronic and complex disease determined by lifestyle behaviors associated with positive energy balance, such as an inappropriate diet and a sedentary lifestyle $(7,8)$. 
The prevalence of obesity has risen all over the world to pandemic proportions during the last 50 years $(9,10)$. Obesity and BMI prevalence vary across different countries according to the steepness of increases, slowing-down, and acceleration periods (11). The global prevalence of obesity almost tripled since 1975 and continues to grow in low- and middle- income countries, although it can be considered as a problem for high-income countries $(10,12,13)$. A systematic review by Rahmani et al. (2015), reported the prevalence of obesity in Iran has increased between 1995 and 2010 (14). Also, Azizi et al. (2005) indicated that the prevalence of obesity was most rapidly rising in the 30 to 40 -year-old group in both men and women in Tehran(15). Furthermore, in a study by Sarrafzadegan et al. on ICS (Isfahan Cohort Study) data (20012007), it was reported that younger individuals gained weight more than older ones(16). In a systematic review in Iran, increased age, urbanization, low educational levels, the female gender, and being married, were indicated as some variables that are associated with inequality in obesity (17).

Due to biological and sociocultural differences, obesity distribution patterns differ by gender and age (18). The risks of many chronic diseases commonly increase with age, which is called aging or longitudinal effect. Also, people born or enter the study of the same age have similar living conditions that may affect their disease risk in a particular way; this is a cohort or crosssectional effect (19). Therefore, decomposition of cohort and aging effects could provide some valuable information. The changes in the risk of obesity were assessed using longitudinal and cross-sectional effects of age, in some studies (20-22). In many research, separation of these two effects of age on obesity is of interest. Estimates of the effect of age from cross-sectional analyses may confound Cohort effects on obesity. Longitudinal assessment is needed to determine the aging effect. In contrast with cross-sectional studies, a longitudinal study is defined as a study in which individuals are measured repeatedly through time. Longitudinal 
studies help us to distinguish changes over time within individuals (longitudinal effect) from differences among people in their baseline levels (cross-sectional effect) $(23,24)$.

A limited number of longitudinal analyses of the prevalence of obesity in population-based samples were done to separate aging and cohort effects and allow simultaneous assessment of aging-related changes and secular trends. In the current study, a longitudinal approach is used to distinguish between cross-sectional and longitudinal effects of age. This study is a large community-based study in a group of 6504 Iranian adults over 35 years of age who live in the central region of Iran. Based on BMI, repeated measurements of obesity were obtained in 2001, 2007, and 2013. A Marginal Logistic Regression (MLR) model was used: 1-To determine whether the prevalence of obesity changes with age in the target population and whether the patterns of change in obesity are the same for women and men. 2-To assess the cross-sectional and longitudinal effects of age. The current study is the first study in Iran that measures the longitudinal and cross-sectional effects of age on obesity, using an advanced statistical modeling approach for longitudinal measurements, the MLR model. This study is benefiting from a population-based cohort study with 12 years of follow-up and is performed among the Iranian adult population. Investigation of prevalence and trend of obesity in a population-based study provides opportunities to target subpopulations who need more care and attention in public health interventions.

\section{Methods}

Study population

Isfahan Cohort Study is a longitudinal ongoing population-based study, including 6504 adults aged over or equal to 35-year-old at the first measurement occasion, from three provenances in the central area of Iran (Isfahan, Arak, and Najafabad), living in urban or rural areas. The participants had been involved in the first survey of the Isfahan Healthy Heart Program (IHHP), 
a community trial for prevention and control of Cardiovascular Diseases (CVDs) $(25,26)$. These three cities were selected due to their population consistency and a smaller number of migrants in comparison with the capital and other cities in Iran. Also, Isfahan is the third-largest city in Iran. The multistage random cluster sampling method was used for sampling these provinces, which represent their society's distribution of age, genders, and residential area (urban/rural). Ethical permission was issued by the Ethics Committee of Isfahan Cardiovascular Research Center. Questionnaires and anthropometric information were collected after obtaining informed written consent in 2001, 2007, and 2013 (25). The number of individuals in the first measurement occasion was 6504, and then during the follow-up, the number decreased to 3284 and 1735 at the second and third phases, respectively. Details of the study design and ICS challenges, which led to a decrease in sample size, were described in previous papers (25-27).

Variables under study

Measurement of height was conducted using a secured metal ruler in barefoot and, weight was measured in light clothing using a calibrated scale (26). BMI was defined as the weight $(\mathrm{Kg})$ divided by height squared $\left(\mathrm{m}^{2}\right)(5)$. A BMI over or equal to 30 indicates obesity, according to the World Health Organization definition (12). Also, waist circumference (WC) (cm) was measured horizontally. It is defined as the smallest circumference below the lowest ribs (26). In our study, although baseline measurements for all individuals were recorded at the same calendar time, age of individuals varied at the entry to the survey. Accordingly, the variations in the prevalence of obesity with age have two potential sources of information. First, the crosssectional (or between-subject) information is about how obesity changes with age in the baseline observations obtained in 2001. Second, since individuals were measured repeatedly through the study time, the longitudinal (or within-subject) information raised. "Baseline Age" was defined as the individual age (year) at baseline and is used to assess the cross-sectional 
effect of age. "Age - Baseline Age" was defined as the years passed since baseline and is used to assess the longitudinal effect of age.

Statistical analysis

The characteristics of the participants at the three measurement occasions $(2001,2007$, and 2013) are presented as a percentage or mean and standard deviation (SD) when appropriate. Considering the outcome as a binary variable (obese or not obese), Marginal Logistic Regression Model was used (equation (1)). The parameters in the model were estimated using Generalized Estimating Equations (GEE) method.

$\log _{e}\left\{\frac{P\left(Y_{i j}\right)=1}{P\left(Y_{i j}\right)=0}\right\}=\beta_{0}+\beta_{1} X_{i j 1}+\cdots+\beta_{P} X_{i j P}$

In equation (1), $Y_{i j}$ denotes the binary response variable for the $i^{t h}$ individual on the $j^{t h}$ occasion. Also, $X_{i j}$ denotes a vector of covariates, associated with the response at each occasion, for each individual. In our study, the main effects include Baseline Age, Age Baseline Age, and Gender. This model considers separate parameters for the longitudinal and cross-sectional effects of age on the binary response and provides an estimation of both effects of age, simultaneously (28). The hypothesis of the equality of the longitudinal and crosssectional effect of age was examined, using the multivariate Wald test for contrasts. SPSS 19 and SAS 9.4, PROC GENMOD were used for the analysis, and the significance level was considered as 0.05 .

\section{Results}


The study population was approximately balanced according to sex (51.3 percent women and 48.7 percent men). The characteristics of the individuals who participated in this study are represented in table 1 by gender and measurement time (2001, 2007 and 2013).

\section{Table 1}

In this study, most of the participants had less than 12 years of education (93.9\% in 2001, 91.7 in 2007, and 88.7 in 2013) and were married (90.6 in 2001, 89.1 in 2007, and 86.3 in 2013). The average age of participants was 50.2 in 2001, 56.1 in 2007, and 60.3 in 2013.

For both men and women, the average BMI and WC were increased over 12 years (2001 2013). Also, women had an average BMI and WC more than men in all three phases. The prevalence of general obesity was calculated in men and women in 2001, 2007, and 2013. According to table 1, the percentages of women classified as obese were 31 in 2001, 39 in 2007, and 44 in 2013, which is more than double the percentages of obese men (13 in 2001, 15 in 2007, and 18 in 2013). In other words, between 2001 and 2013, the prevalence of obesity raised from $13 \%$ to $18 \%$ in men and from $31 \%$ to $44 \%$ in women. In the total population, the prevalence of obesity raised from $22 \%$ in 2001 to $31 \%$ in 2013.

The percentages of individuals indicated as obese at each of the three measurement occasions for the Baseline Age groups of 35-44, 45-54, 55-64, and $\geq 65$ years in men and women and longitudinal changes in the prevalence of obesity over the follow-up period, are displayed in table 2. These percentages were reported based on the available data at each occasion in each age-gender group.

\section{Table 2}

In table 2, the rates of obesity for women are higher in all ages and on all three occasions. Also, we can identify the cross-sectional effect of age, by considering obesity prevalence in each year of measurement and compare it over different Baseline Age groups. 
The associations between Baseline Age and prevalence of obesity in each occasion by gender are shown in Figure 1 in an additional file [see Additional file 1]. Growth in the prevalence of obesity from 35 to 55 Baseline Age groups can be identified, followed by a decrease for higher Baseline Age in all three phases and both genders, except for females in 2013, which had a peak in obesity prevalence in 55-65 Baseline Age groups. In other words, there is approximately an elevated probability for obesity for people in 35-55 Baseline Age groups, followed by a modest decrease in probability for people who were in the older age group $(\geq 55)$. Also, to identify the longitudinal effect of age, we can consider each Baseline Age group separately and compare the prevalence of obesity in different measurement times. The associations between aging and the probability of obesity by Baseline Age groups are shown in Figure 2 in an additional file [see Additional file 2]. It shows obesity prevalence for the same people over time. Obesity prevalence for each group raised with time through the study except for Baseline Age $\geq 65$ in both men and women that means obesity prevalence increased with aging for individuals in Baseline Age groups 35-65. For women in higher than 65 Baseline Age groups, there is a moderate decrease after six years, and then the probability of obesity tending to be almost constant by aging. Also, for men in Baseline Age groups $\geq 65$, there was a decrease after six years and then a moderate increase after twelve years.

According to the rates of obesity in table 2 and Figure 1 which show the curvilinear effect of age on the rate of obesity and higher rates of obesity for women of all ages, an MLR model including gender, linear and quadratic age, and the gender by age interactions was fitted (equation (2)). Individuals were classified as obese $\left(Y_{i j}=1\right)$ or not obese $\left(Y_{i j}=0\right)$.

$$
\begin{aligned}
\log _{e}\left\{\frac{P\left(Y_{i j}\right)=1}{P\left(Y_{i j}\right)=0}\right\}= & \beta_{0}+\beta_{1} \text { Gender }_{i}+\beta_{2} \text { Age }_{i j}+\beta_{3} \text { Age }_{i j}{ }^{2}+\beta_{4} \text { Gender }_{i} \times \text { Age }_{i j} \\
& +\beta_{5} \text { Gender }_{i} \times \text { Age }_{i j}{ }^{2} .
\end{aligned}
$$


In this model, the gender by age interactions was not significant ( $\mathrm{P}$-value $=0.45$ and 0.35 for gender by age and gender by age squared respectively and the multivariate Wald test, $H_{0}: \beta_{4}=$ $\beta_{5}=0$ Chi-Square $=2.75, \mathrm{DF}=2, \mathrm{P}$-value $=0.25$ ), so the interaction terms were omitted from the model. It shows there is no significant difference in changes (trend) of log OR of obesity between men and women. The results are presented in an additional file [see Additional file 3 Table 1]. Also, the significant coefficients of age and age squared showed that the log OR of obesity changes with age curvilinearly (P-value<0.0001). Next, to assess the longitudinal and cross-sectional effects of age on log OR of obesity, another MLR model including linear and quadratic effects of the Baseline Age and Age - Baseline Age $\left(A g e_{i 1}\right.$ and $A g e_{i j}-A g e_{i 1}$, respectively), was fitted. According to descriptive statistics and the coefficient in the model, the quadratic effect of Age - Baseline Age had a little and negligible effect on the prevalence of obesity $(\beta=-0.0006$ and $\operatorname{EXP}(\beta)=0.999)$. Therefore, to simplify the model, we preferred to omit this effect also, and the model in equation (3) was considered.

$\log _{e}\left\{\frac{P\left(Y_{i j}\right)=1}{P\left(Y_{i j}\right)=0}\right\}=\beta_{0}+\beta_{1}$ Gender $_{i}+\beta_{2} A g e_{i 1}+\beta_{3} A g e_{i 1}{ }^{2}+\beta_{4}\left(A g e_{i j}-A g e_{i 1}\right)$.

In equation (3), the linear combination of $\beta_{2}$ and $\beta_{3}$ indicates the cross-sectional effect of age. They describe how the log odds ratio (OR) of obesity changes with age at baseline. On the other hand, $\beta_{4}$ indicates the longitudinal effect of age because it represents how within-subject differences in the log OR of obesity are associated with within-subject changes in age $(23,29)$. Then, using the multivariate Wald test for contrasts, the hypothesis of equality of crosssectional and longitudinal effects was examined. The hypothesis is equivalent to equality of the coefficients of (Baseline Age) with (Age- Baseline Age) or $H_{0}: \beta_{2}=\beta_{4}$ in equation (3). 
The Wald test was significant (Chi-Square $=12.63, \mathrm{DF}=1, \mathrm{P}$-value $<0.0004)$, which means the longitudinal effect is not equal to the cross-sectional effect of age and it shows the effect of aging on the odds of obesity.

\section{Table 3}

In table 3, Alpha1, Alpha2, and Alpha3 are pairwise log OR among measurement occasions. The pairwise log OR between adjoining occasions are almost 2.9 and 3.5, which indicate that the OR for the within-subject association is about 18 and 33 , respectively (or $\mathrm{e}^{2.9}$ and $\mathrm{e}^{3.5}$ ). Therefore, there is a severe positive association among the indicators of obesity status at all measurement occasions.

The significance coefficient of sex shows that log OR of obesity is different between women and men. By controlling the age effects (longitudinal and cross-sectional effects), odds of being obese for women is approximately $\exp (1.15)=3.17$ times the odds of being obese for men on average (Gender=1 was considered for men in the dataset and reference group in the model). Hence, women are more likely to be obese (almost three times) than men.

For cross-sectional and longitudinal effects of age, the results of the model are compatible with the results of descriptive statistics. According to table 3, the linear combination of the significant coefficients for Baseline Age and Baseline Age squared in the model can be interpreted as the overall Baseline Age group (or cross-sectional) effect controlling for the longitudinal effect of age and sex (20). The log OR of obesity alters curvilinearly with Baseline Age. According to the rates of obesity in table 2, we understand there is an increase in the prevalence of obesity for individuals with Baseline Ages from 35 to 54 and a decline thereafter. Moreover, the significant coefficients for (Age - Baseline Age) in the model can be interpreted as the longitudinal effect controlling for the cross-sectional effect of age and gender (20). The odds of obesity changes over time within individuals. In other words, the odds of obesity across one's life course, on average, has about 3\% increase by each year aging, regardless of the 
Baseline Age group which the individuals are come from $(\operatorname{EXP}(0.0295)=1.029)$. According to table 2, we can conclude that obesity prevalence for each Baseline Age group raised with time during the study for both men and women except for Baseline Age group $\geq 65$. In other words, obesity prevalence increased with aging for individuals in Baseline Age group 35-65.

Also, according to the estimated coefficients and ORs in Table 3, in this study, the crosssectional effect of age was more considerable than the longitudinal effect of it. In other words, the Baseline Age was more strongly associated with odds of obesity than aging (linear combination of 0.1266 and -0.0013 for cross-sectional effect vs. 0.0295 for longitudinal effect).

\section{Discussion}

The goals in this study were first, to determine whether the probability of obesity changed with age and whether patterns of change in obesity were the same for women and men, and second, to assess the cross-sectional and longitudinal effects of age. Our study was a large communitybased study in a group of 6504 Iranian adults over 35 years of age who live in the central region of Iran. In our study, the prevalence of obesity (in total) increased from $22 \%$ in 2001 to $31 \%$ in 2013, while according to the WHO report, between 2000 and 2016, in all WHO regions and income groups, the global prevalence of obesity raised from $9 \%$ in 2000 to $13 \%$ in 2016 (12). It shows that the prevalence of obesity in Iran was higher than the global prevalence of obesity (almost double) in the same period. Also, the amount of increase in the prevalence of obesity in Iran was more than global prevalence (in Iran, an 8\% increase in 12 years; global average, 4\% in 16 years). This massive increase is almost as large as the increase in the American population, which increased from 20\% in 2000 to $29 \%$ in 2016 (12). Also, the result of our study showed that, between 2001 and 2013, the prevalence of obesity increased from $13 \%$ to $18 \%$ (5 percentage points increase) in adult men and from $31 \%$ to $44 \%$ (13 percentage points increase) in adult women, while between 1975 and 2014, the global prevalence of obesity raised from $3.2 \%$ to $10.8 \%$ in men and from $6.4 \%$ to $14.9 \%$ in women (1). Shifts in diets and 
eating behavior which include energy-dense foods, high in sugars and fat, higher consumption of red meat, salt, and saturated fatty acids among individuals because of the increasing economic status, and less physical activity due to the sedentary work styles, types of transportation and changes in lifestyle are combining to increase the possibility of becoming obese (4). This rate of increase is also reported by other studies $(1,6,15,16,30)$. In both developed and developing countries and specifically in middle-east countries, the prevalence of obesity is rising at alarming rates $(1,4,13)$.

Additionally, in our study, using the GEE model and descriptive analysis, the odds of being obese for women is more than for men. It is approximately $\exp (1.15)=3.158$ times of odds of being obese for men on average and by controlling the age effects (aging and cross-sectional effects). In other words, we can conclude that women are more likely to be obese (almost three times) than men. In another national study in Iran (the National Health Survey), the adjusted odds of obesity for women among Iranian adults aged 20-59 years were 2.4, 2.35, and 1.58 times of odds of being obese for men in 2000, 2007, and 2011, respectively (31).

In our study, for both groups of men and women, the average BMI and WC were raised over 12 years $(2001$ - 2013), but women had a higher average BMI and WC than men in all three phases. Among women, lower physical activity level, pregnancy, menopause, a higher rate of depression, lack of employment, lower socioeconomic status, lower educational level, and gender differences in food intake may be some of the causes for the higher prevalence of obesity in comparison with men $(30,32-34)$. Iranian women pay less attention to their body shape in comparison to European and Oceanic women and also, less information and knowledge about weight loss may be another cause (15)

Considerable regional differences in BMI alters through time were reported in different studies. In Southern Asia (Bangladesh, Bhutan, India, Nepal, and Pakistan), in southeast Asia ( Malaysia, Indonesia, Philippines, Sri Lanka, Vietnam, and Thailand), in the Caribbean (Cuba, 
Belize, Jamaica, Puerto Rico, and the Dominican Republic), and in southern Latin America ( Brazil, Argentina, Paraguay, Uruguay, and Chile), an accelerated rise in BMI was reported (1). In our study, applying an MLR model, it was shown that there is a difference between the crosssectional effect and longitudinal effect of age on obesity prevalence in the target population, controlling for the gender effect. In this study, the cross-sectional effect of age was more considerable than the longitudinal effect of it. In other words, the Baseline Age was more severely associated with the odds of obesity than aging. The cross-sectional effect of age shows differences among people in their Baseline Age. There was a growth in the prevalence of obesity for individuals with Baseline Ages from 35 to 55 and a decline thereafter. In other studies, it is reported that Iranian adults gain weight until the age of about 50-60 years old, and after this age, BMI tends to decrease. The results of our study on the association between the probability of obesity and age are close to some of the other studies(16, 17, 35-38). Previous studies reported that BMI had a tendency to be higher in individuals with middle-aged in comparison with young adults and also tended to be constant or decrease in older individuals. Also, in a study in the United States of America (USA), NHANES-III, BMI had tendency to go up for ages 20 to 29 years till ages 50 to 59 years, and after the age of 59, BMI tended to decline (38). In another study in the USA, an independent cross-sectional effect of age on obesity was reported and also, a higher probability for obesity in younger Baseline Age groups in comparison to older individuals after controlling the aging effects were observed (21). With increasing age, there are alterations in food intake, energy consumption, and appetite besides bone and muscle loss that affect body composition (30).

Also, the longitudinal effect of age shows changes over time within individuals or changes across one's life course, regardless of the Baseline Age group for individuals. In our study, obesity prevalence for each group increased with time within the study for both men and women except for Baseline Age group $\geq 65$ in women. It can be concluded that obesity 
prevalence increasing with aging for individuals in Baseline Age groups 35-65. For women in more than 65 Baseline Age groups, there is a moderate decrease after six years, and then the probability of obesity tending to be almost constant after twelve years. Also, for men in Baseline Age group $\geq 65$, there was a decrease after six years and then a moderate increase after twelve years. Odds of obesity across one's life course, on average, have about a 3\% increase by each year aging, regardless of the membership in each of Baseline Age groups. Most studies confirmed a severe aging effect on the high prevalence of obesity in middle age. For example, in a study in the USA, a shape similar to an inverted $U$ for age effect on obesity and a lifted probability for obesity as an individual transition from young adulthood to midlife and a moderate decrease probability as one approaches the end of life was reported (21). Several factors might be responsible for the reduction in the old Baseline Age group; the most possible is the decrease in appetite and abdominal obesity in the elderly. Frequent medical check-up and treatment in older people may be another cause for this result. Moreover, older people may not be interested in modern lifestyles, such as the changes in dietary preferences $(39,40)$.

This study had several strengths, including its large sample size from a longitudinal ongoing community-based study of adults living in urban or rural areas from three providences in the central region of Iran (Isfahan Healthy Heart Program). Furthermore, in this study, we assessed both longitudinal effect (aging effect) and cross-sectional (Baseline Age effect) of age on obesity prevalence. However, despite the unique coverage of our study in comparison with other studies in Iran, the sample represented the population of central parts of Iran, and it limits the generalizability of our findings to the entire country. Also, our study assessed the longitudinal effect based on only three measurements; using data with more repeated measures for individuals, the accuracy of results will improve. Also, in our study, the goal was only to assess the relationship between obesity prevalence with the cross-sectional and longitudinal effect of age in men and women, but other confounders were not considered. 


\section{Conclusions}

Obesity is a severe public health issue among Iranian adults. Available data in our study indicates a high rate of obesity and its fast growth, especially in adults age 35-55 and women. Also, Baseline Age was more strongly associated with the risk of obesity than aging. Considering both cross-sectional and longitudinal effects of age, helps us to understand the effect of age on obesity better and to identify the related factors. It is necessary to determine the factors contributing to the growth in the prevalence of obesity.

\section{List of Abbreviations}

Noncommunicable Diseases (NCDs), World Health Organization (WHO), Body Mass Index (BMI), Isfahan Healthy Heart Program (IHHP), The Isfahan Cohort Study (ICS), Waist Circumference (WC), Standard Deviation (SD), Cardiovascular Diseases (CVDs), Generalized Estimating Equations (GEE), Odds Ratio (OR), United States of America (USA), Marginal Logistic Regression (MLR)

\section{Declarations}

Ethics approval and consent to participate

Ethical approval was obtained from the Ethics Committee of Isfahan Cardiovascular Research Center (ICRC), a World Health Organization-collaborating center, and all subjects gave written informed consent.

Consent for publication

Not applicable

Availability of data and materials

The data that support the findings of this study are available on request from the corresponding author MS. The data are not publicly available due to privacy.

Competing interests

The authors declare that they have no competing interests

Funding 
No funding

Authors' contributions

MR developed and wrote the manuscript. AK, FZ, MS screened the literature, designed, edited, and approved the final version. All authors read and approved the final manuscript.

Acknowledgments

The authors state their appreciation to the Isfahan Cardiovascular Research Institute personnel.

Authors' information

MR, Ph.D. candidate, Department of Biostatistics, Faculty of Medical Sciences, Tarbiat Modares University, P.O. Box 14115111, Tehran, Iran, Email: mhsrafiee@modares.ac.ir

FZ, Professor of Biostatistics, Department of Biostatistics, Proteomics Research Center, School of Allied Medical Sciences, Shahid Beheshti University of Medical Sciences, P.O. Box: 1971653313, Tehran, Iran, Phone: +98 21 22439982, Email: f_zayeri@ sbmu.ac.ir

Corresponding authors:

AK, Professor of Biostatistics and head of the Department of Biostatistics, Faculty of Medical Sciences, Tarbiat Modares University, PO. Box 14115111, Tehran, Iran, Phone: +98 21 82883875, Email: kazem_an@modares.ac.ir

MS, Professor of Cardiology and Head of the Cardiac Rehabilitation Research Center, Isfahan Cardiovascular Research Institute, Isfahan University of Medical Sciences, PO. Box 58388994, Isfahan, Iran, Phone: +98 3136115210 , Email: m_sadeghi@crc.mui.ac.ir

\section{References:}

1. Blüher M. Obesity: global epidemiology and pathogenesis. Nature Reviews Endocrinology. 2019;15(5):288.

2. Forouzanfar MH, Afshin A, Alexander LT, Anderson HR, Bhutta ZA, Biryukov S, et al. Global, regional, and national comparative risk assessment of 79 behavioural, environmental and occupational, and metabolic risks or clusters of risks, 1990-2015: a systematic analysis for the Global Burden of Disease Study 2015. The lancet. 2016;388(10053):1659-724.

3. Collaboration PS. Body-mass index and cause-specific mortality in 900000 adults: collaborative analyses of 57 prospective studies. The Lancet. 2009;373(9669):1083-96.

4. World Health Organization. https://www.who.int/nmh/publications/ncd-progress-monitor2017/en/. Accessed 12 March 2020.

5. Sadeghi M, Talaei M, Gharipour M, Oveisgharan S, Nezafati P, Dianatkhah $M$, et al. Anthropometric indices predicting incident hypertension in an Iranian population: The Isfahan cohort study. Anatolian journal of cardiology. 2019;22(1):33.

6. Emamian MH, Fateh M, Hosseinpoor AR, Alami A, Fotouhi A. Obesity and its socioeconomic determinants in Iran. Economics \& Human Biology. 2017;26:144-50.

7. de Munter Js, Tynelius P, Magnusson C, Rasmussen F. Longitudinal analysis of lifestyle habits in relation to body mass index, onset of overweight and obesity: results from a large population-based cohort in Sweden. Scandinavian journal of public health. 2015;43(3):236-45.

8. Seidell JC, Halberstadt J. The global burden of obesity and the challenges of prevention. Annals of Nutrition and Metabolism. 2015;66(Suppl. 2):7-12. 
9. Yanovski JA. Trends in underweight and obesity-scale of the problem. Nature Reviews Endocrinology. 2018;14(1):5-6.

10. Collaboration NRF. Worldwide trends in children's and adolescents' body mass index, underweight, overweight and obesity, in comparison with adults, from 1975 to 2016: a pooled analysis of 2, 416 population-based measurement studies with 128.9 million participants. Lancet. 2017;390:2627-42.

11. Collaboration NRF. Trends in adult body-mass index in 200 countries from 1975 to 2014: a pooled analysis of 1698 population-based measurement studies with $19 \cdot 2$ million participants. The Lancet. 2016;387(10026):1377-96.

12. World Health Organization. https://www.who.int/nmh/publications/ncd-profiles-2018/en/. Accessed 12 March 2020.

13. Kilpi F, Webber L, Musaigner A, Aitsi-Selmi A, Marsh T, Rtveladze K, et al. Alarming predictions for obesity and non-communicable diseases in the Middle East. Public health nutrition. 2014;17(5):1078-86.

14. Rahmani A, Sayehmiri K, Asadollahi K, Sarokhani D, Islami F, Sarokhani M. Investigation of the prevalence of obesity in Iran: a systematic review and meta-analysis study. Acta Medica Iranica. 2015:596-607.

15. Azizi F, Azadbakht L, Mirmiran P. Trends in overweight, obesity and central fat accumulation among Tehranian adults between 1998-1999 and 2001-2002: Tehran lipid and glucose study. Annals of nutrition and metabolism. 2005;49(1):3-8.

16. Sarrafzadegan N, Talaei $M$, Sadeghi $M$, Mohammadifard N, Lotfizadeh Dehkordi $M$, Esmaillzadeh A, et al. Determinants of weight change in a longitudinal study of Iranian adults: Isfahan Cohort Study. Archives of Iranian medicine. 2014;17(8):539-44.

17. Djalalinia S, Peykari N, Qorbani M, Larijani B, Farzadfar F. Inequality of obesity and socioeconomic factors in Iran: a systematic review and meta-analyses. Medical journal of the Islamic Republic of Iran. 2015;29:241.

18. Yamada G, Castillo-Salgado C, Jones-Smith JC, Moulton LH. Differences in magnitude and rate of change in adult obesity distribution by age and sex in Mexico, Colombia and Peru, 2005-2010. Public health nutrition. 2019;22(4):757-63.

19. Diouf I, Charles MA, Ducimetière $P$, Basdevant A, Eschwege E, Heude B. Evolution of obesity prevalence in France: an age-period-cohort analysis. Epidemiology (Cambridge, Mass). 2010;21(3):360.

20. Jaacks LM, Gordon-Larsen P, Mayer-Davis EJ, Adair LS, Popkin B. Age, period and cohort effects on adult body mass index and overweight from 1991 to 2009 in China: the China Health and Nutrition Survey. International journal of epidemiology. 2013;42(3):828-37.

21. An R, Xiang X. Age-period-cohort analyses of obesity prevalence in US adults. Public Health. 2016;141:163-9.

22. Hosseini M, Kelishadi R, Baikpour M, Ataei N, Qorbani M, Yousefifard M, et al. Age-periodcohort analysis of obesity and overweight in Iranian children and adolescents. International journal of endocrinology and metabolism. 2017;15(4).

23. Diggle P, Diggle PJ, Heagerty P, Liang K-Y, Heagerty PJ, Zeger S. Analysis of longitudinal data: Oxford University Press; 2002.

24. Mason WM, Fienberg SE. Introduction: Beyond the identification problem. Cohort analysis in social research: Springer; 1985. p. 1-8.

25. Sarrafzadegan N, Baghaei A, Sadri G, Kelishadi R, Malekafzali H, Boshtam M, et al. Isfahan healthy heart program: Evaluation of comprehensive, community-based interventions for noncommunicable disease prevention. Prevention and control. 2006;2(2):73-84.

26. Sarrafzadegan N, Talaei M, Sadeghi M, Kelishadi R, Oveisgharan S, Mohammadifard N, et al. The Isfahan cohort study: rationale, methods and main findings. Journal of human hypertension. 2011;25(9):545. 
27. Sarrafzadegan N, Hassannejad R, Roohafza H, Sadeghi M, Talaei M, Oveisgharan S, et al. A 10year Isfahan cohort on cardiovascular disease as a master plan for a multi-generation noncommunicable disease longitudinal study: methodology and challenges. Journal of human hypertension. 2019;33(11):807-16.

28. Fitzmaurice GM, Laird NM, Ware JH. Applied longitudinal analysis: John Wiley \& Sons; 2012.

29. Fitzmaurice G, Davidian M, Verbeke G, Molenberghs G. Longitudinal data analysis: CRC Press; 2008.

30. Bakhshi E, Etemad K, Seifi B, Mohammad K, Biglarian A, Koohpayehzadeh J. Changes in obesity odds ratio among Iranian adults, since 2000: Quadratic inference functions method. Computational and mathematical methods in medicine. 2016;2016.

31. Bakhshi E, Seifi B, Biglarian A, Mohammad K. Factors associated with obesity in Iranian elderly people: results from the national health survey. BMC research notes. 2011;4(1):538.

32. Ghassemi H, Harrison G, Mohammad K. An accelerated nutrition transition in Iran. Public health nutrition. 2002;5(1a):149-55.

33. Azizi F, Allahverdian S, Mirmiran P, Rahmani M, Mohammadi F. Dietary factors and body mass index in a group of Iranian adolescents: Tehran lipid and glucose study-2. International journal for vitamin and nutrition research. 2001;71(2):123-7.

34. Torrance GM, Hooper MD, Reeder BA. Trends in overweight and obesity among adults in Canada (1970-1992): evidence from national surveys using measured height and weight. International journal of obesity. 2002;26(6):797-804.

35. Asgari F, Biglarian A, Seifi B, Bakhshi A, Miri HH, Bakhshi E. Using quadratic inference functions to determine the factors associated with obesity: findings from the STEPS Survey in Iran. Annals of epidemiology. 2013;23(9):534-8.

36. Song PXK, Jiang Z, Park E, Qu A. Quadratic inference functions in marginal models for longitudinal data. Statistics in medicine. 2009;28(29):3683-96.

37. Qu A, Li R. Quadratic inference functions for varying-coefficient models with longitudinal data. Biometrics. 2006;62(2):379-91.

38. Stevens J, Jones DW, Arnett D. Associations of aging and birth cohort with body mass index in a biethnic cohort. Obesity research. 2003;11(3):426-33.

39. Hickson M. Malnutrition and ageing. Postgraduate medical journal. 2006;82(963):2-8.

40. Ahmed F, Waslien C, Al-Sumaie MA, Prakash P. Secular trends and risk factors of overweight and obesity among Kuwaiti adults: National Nutrition Surveillance System data from 1998 to 2009. Public Health Nutrition. 2012;15(11):2124-30.

\section{Tables}

Table 1. Characteristics of the Study Participants in 2001, 2007, and 2013 by Gender

\begin{tabular}{|c|c|c|c|c|c|c|c|c|c|c|}
\hline & \multicolumn{3}{|c|}{2001} & \multicolumn{3}{|c|}{2007} & \multicolumn{3}{|c|}{2013} \\
\hline & & Female & Male & Total & Female & Male & Total & Female & Male & Total \\
\hline \multicolumn{2}{|c|}{ Number of Participants } & 3336 & 3168 & 6504 & 1680 & 1604 & 3284 & 881 & 854 & 1735 \\
\hline \multirow[t]{3}{*}{ Education (\%) } & Level $1^{\dagger}$ & 80.7 & 63.4 & 72.3 & 76.7 & 59.1 & 68.1 & 68.3 & 49.8 & 59.1 \\
\hline & Level $2^{\dagger}$ & 16.7 & 26.8 & 21.6 & 19.7 & 27.6 & 23.6 & 24.9 & 34.3 & 29.6 \\
\hline & Level $3^{\dagger}$ & 2.5 & 9.6 & 6 & 2.9 & 12.5 & 7.6 & 5.5 & 15.4 & 10.4 \\
\hline Married(\%) & & 83.5 & 98.1 & 90.6 & 80.8 & 97.8 & 89.1 & 77.3 & 95.4 & 86.3 \\
\hline Age $^{*}$ & & $\begin{array}{c}49.7 \\
(11.5)\end{array}$ & $\begin{array}{l}50.7 \\
(12)\end{array}$ & $\begin{array}{c}50.2 \\
(11.7)\end{array}$ & $\begin{array}{c}55.5 \\
(10.2)\end{array}$ & $\begin{array}{c}56.7 \\
(10.8)\end{array}$ & $\begin{array}{c}56.1 \\
(10.5)\end{array}$ & $\begin{array}{c}60 \\
(9.6)\end{array}$ & $\begin{array}{l}60.6 \\
(9.8)\end{array}$ & $\begin{array}{l}60.3 \\
(9.7)\end{array}$ \\
\hline BMI* & & $\begin{array}{l}27.9 \\
(5.1)\end{array}$ & $\begin{array}{c}24.5 \\
(4)\end{array}$ & $\begin{array}{l}26.7 \\
(4.7)\end{array}$ & $\begin{array}{l}28.7 \\
(4.6)\end{array}$ & $\begin{array}{l}26.1 \\
(3.8)\end{array}$ & $\begin{array}{l}27.7 \\
(4.4)\end{array}$ & $\begin{array}{l}29.4 \\
(4.8)\end{array}$ & $\begin{array}{l}26.5 \\
(4.3)\end{array}$ & $\begin{array}{c}28 \\
(4.8)\end{array}$ \\
\hline
\end{tabular}




\begin{tabular}{lccccccccc} 
WC $^{*}$ & 96.4 & 92.3 & 94.4 & 96.2 & 93.8 & 95 & 98.3 & 97.4 & 97.8 \\
& $(13.4)$ & $(12)$ & $(12.9)$ & $(12.2)$ & $(25)$ & $(19.5)$ & $(12.2)$ & $(10.6)$ & $(11.4)$ \\
\hline Obesity (\%) & 31 & 13 & 22 & 39 & 15 & 27 & 44 & 18 & 31 \\
\hline
\end{tabular}

*Data are presented as Mean (SD).

'Level 1, Illiterate or elementary school; Level 2, intermediate school or high school; Level 3, university degree

Table 2. Prevalence of general obesity 2001, 2007, and 2013 by Gender

\begin{tabular}{|c|c|c|c|c|c|c|c|c|c|}
\hline \multirow{2}{*}{$\begin{array}{c}\text { Baseline Age } \\
\text { categories }\end{array}$} & \multicolumn{3}{|c|}{2001} & \multicolumn{3}{|c|}{2007} & \multicolumn{3}{|c|}{2013} \\
\hline & Female & Male & Total & Female & Male & Total & Female & Male & Total \\
\hline $35-44$ & 31.1 & 11.4 & 21.7 & 40.5 & 13.4 & 27.9 & 46.3 & 17 & 32.1 \\
\hline $45-54$ & 35.5 & 15.5 & 25.9 & 41.8 & 19.3 & 30.7 & 42.7 & 21.5 & 31.8 \\
\hline $55-64$ & 33.7 & 14.3 & 23.9 & 37.8 & 14.5 & 25.1 & 47.8 & 17.2 & 30.8 \\
\hline$\geq 65$ & 24.5 & 10.8 & 17.2 & 21.9 & 9.3 & 15.4 & 22.4 & 16.4 & 19.7 \\
\hline Total & 31.4 & 13 & 22.4 & 38.5 & 14.8 & 27 & 43.6 & 18.3 & 31 \\
\hline
\end{tabular}

Data are presented as percentage.

Obesity, BMI $\geq 30$ (World Health Organization recommendation)

Table 3. Results from MLR Model to assess Gender and longitudinal and cross-sectional Age effects on obesity

\begin{tabular}{|c|c|c|c|c|c|}
\hline Parameter & Estimate $(\beta)$ & Standard Error & P-value & OR & 95\% CI OR \\
\hline Intercept & -4.9664 & 0.6952 & $<0.001$ & 0.006 & $(0.001,0.027)$ \\
\hline Gender (Female) & 1.1546 & 0.0583 & $<0.001$ & 3.172 & $(2.830,3.555)$ \\
\hline Baseline Age & 0.1266 & 0.0269 & $<0.001$ & 1.134 & $(1.076,1.196)$ \\
\hline Baseline Age squared & -0.0013 & 0.0003 & $<0.001$ & 0.998 & $(0.998,0.999)$ \\
\hline Age- Baseline Age & 0.0295 & 0.0038 & $<0.001$ & 1.029 & $(1.022,1.037)$ \\
\hline Alpha1 & 2.9046 & 0.1050 & $<0.001$ & 18.257 & $(14.861,22.432)$ \\
\hline Alpha2 & 2.6129 & 0.1421 & $<0.001$ & 13.638 & $(10.323,18.018)$ \\
\hline Alpha3 & 3.5040 & 0.1754 & $<0.001$ & 33.248 & $(23.575,46.885)$ \\
\hline
\end{tabular}

$\mathrm{QIC}=12070.3512 ; \mathrm{QICu}=12066.9720$

\section{Additional Files}

File name: Additional file 1.pdf

File format: pdf 
Title of data: Figure 1. Cross-sectional effect of age on prevalence of obesity in men and women

File name: Additional file 2.pdf

File format: pdf

Title of data: Figure 2. Longitudinal effect of age on prevalence of obesity in men and women

File name: Additional file 3.pdf

File format: pdf

Title of data: Table 1. Results from MLR Model to assess Gender and Age effects on obesity 